\title{
Experimental antithrombotic effect of potatoes harvested in the autumn
}

\author{
Junichiro Yamamoto ${ }^{1^{*}}$, Megumi Masuda ${ }^{1}, K$ anae Hyodo ${ }^{1}$, Masahiro Iwasaki $^{2}$ \\ ${ }^{1}$ Laboratory of Physiology, Faculty of Nutrition, Cooperative Research Center of Life Sciences, Kobe Gakuin University, Kobe, \\ Japan; ${ }^{*}$ Corresponding Author: yamamoto@nutr.kobegakuin.ac.jp \\ ${ }^{2}$ Division of Metabolism and Clinical Nutrition, Kansai Electric Power Hospital, Osaka, Japan
}

Received 2 December 2011; revised 26 December 2011; accepted 13 January 2012

\begin{abstract}
Prevention of arterial thrombotic diseases has a high priority in developed countries. While an inappropriate diet is known to enhance the risk for acute thrombotic events, a regular diet with proven antithrombotic effects might be beneficial in the prevention of such diseases. The present study is part of a series of investigations aimed to assess the possible antithrombotic activity of various fruits and vegetables. Previously we demonstrated antithrombotic effect of specific potato varieties harvested in the spring. The present study aimed to test seven varieties of potatoes, which were harvested in the autumn. The in vitro Global Thrombosis Test (GTT) was used for selection, which was then followed by the He-Ne laser-induced in vivo thrombosis test in mice treated with potato filtrates orally. We have shown that all seven potato varieties showed some antithrombotic effect and the heat-resistant effect of four varieties was highly significant. Our present findings add further components to a diet containing fruits and vegetables with antithrombotic effect.
\end{abstract}

Keywords: Cardiovascular Disease; Stroke; Antithrombotic Activity; Global Thrombosis Test (GTT); Vegetables

\section{INTRODUCTION}

The prevention of "lifestyle-related atherothrombotic diseases" such as myocardial infarction and stroke has become an important and urgent social task in many developed countries. Several studies provided clear evidence that an inappropriate diet such as the Western-style high fat diet plays a causative role in the pathogenesis and clinical outcome of thrombotic diseases [1].

The so-called French Paradox and red wine hypothesis has prompted many laboratory studies on finding antithrombotic fruits and vegetables [2,3]. Epidemiological studies provided evidence that intake of fresh fruits and vegetables could help to prevent cardiovascular diseases and stroke [4-7].

Platelets play a pivotal role in arterial thrombotic diseases. Platelet function in vitro is commonly assessed by platelet aggregometry, which measures platelet aggregation induced by various chemical agonists. In clinical practice, different point-of-care platelet function tests are in use. The shortcoming of all these test is the use of citrateanticoagulated blood sample. Citrate anticoagulation is known to distort platelet reactivity, compared to in vivo [8-10]. In this study we employed the Global Thrombosis Test, which uses native (non-anticoagulated) blood and shear stress as platelet agonist [11]. By these, this test is much more physiological than those mentioned above. [11-13].

We have demonstrated that shear-induced platelet reactivity in vitro test (GTT) correlate significantly with the helium-neon (He-Ne) laser-induced in vivo thrombosis tests in mice $[14,15]$. Therefore, these tests were regarded useful in classifying varieties of fruits and vegetables into subgroups with or without antithrombotic/thrombolytic activities.

In the present study, potato varieties harvested in autumn were tested for possible antithrombotic effect first in vitro and then in orally treated animals, in vivo.

\section{MATERIALS AND METHODS}

\subsection{Animals}

Male Wistar ST rats older than 13 weeks old were purchased one week before use (Japan SLC Co. Ltd., Hamamatsu, Japan). Rats were fed a standard solid chow (MF, Oriental Yeast Co. Ltd., Osaka, Japan). Animals were allowed tap water ad libitum, and were maintained in compliance with the "Guiding Principles for the Care and Use of Animals in the field of Physiological Sciences," published by Physiological Society of Japan. The protocol was approved by the Animal Experiment Committee of Kobe Gakuin University. Animals were sacrificed after the test 
by using Somnopentyl.

\subsection{Potatoes}

Seven varieties of autumn harvested potatoes: Dejima, Cherie, Corolle, Cynthia, Sassy, Chelsea and Nishiyutaka were purchased.

\subsection{Preparation of Potato Filtrates}

About six potatoes per one variety were graded at room temperature. The juice was centrifuged $(3000 \mathrm{rpm}, 15$ $\min , 4^{\circ} \mathrm{C}$ ) and the supernatant was filtered (FP30/5.0 CN-S, 5.0 $\mu \mathrm{m}$, Whatman PLC, Kent, UK). The clear filtrates were stored at $-80^{\circ} \mathrm{C}$ before use.

\subsection{Heat Stability}

Having kept in boiling water for 10 min, filtrates were cooled to room temperature and after removing any precipitate formed, were tested by GTT.

\subsection{In Vitro Assessment of Platelet Reactivity and Spontaneous Thrombolytic Activity by the Global Thrombosis Test (GTT)}

The technique has been described in detail elsewhere [14-16]. Figures 1 and 2 illustrate the principles of the technique. A flat segment created along the inner wall of a conical plastic tube forms the basis of the technique. When perfectly round steel ball-bearings are placed into the conical tube, the flat segment prevents the spheres from occluding the lumen. Blood is added, which flows through the narrow gaps between the inner surface of the test tube and the balls and exits in droplets into an adjacent collecting tube. The latter is trans-illuminated by a light emitter, and a sensor opposite the light source generates a signal whenever a drop of blood interrupts the light path. In essence, the instrument detects the time interval $(\mathrm{d}, \mathrm{sec})$ between consecutive blood drops. At the start, blood flow is rapid and hence (d) is small. Subsequently, the flow rate gradually decreases because of thrombi formation in the gaps and hence (d) increases. When the actual (d) exceeds 15 seconds (occlusion-d), the instrument displays "Occlusion Time (OT)", which is the time elapsed from the detection of the first drop of blood until (occlusion-d). Later, flow is completely arrested by the formed thrombi. Eventually, due to thrombolysis, flow is restored as indicated by the further detection of blood droplets. There is also an arbitrarily pre-set (d) (200 seconds) to allow stabilization of the formed thrombi (lysis-d). When (d) between the last drop before and the first drop after occlusion exceeds this (lysis-d), the instrument displays "Lysis Time (LT)". Increased or decreased OT values indicate inhibition or enhancement of platelet reactivity, respecti- vely. Increased or decreased LT values indicate inhibition or enhancement of spontaneous (endogenous) thrombolysis, respectively. Measurements were made six times (n $=6$ ) in each sample.

Animals were fasted overnight but allowed water ad libitum. Blood was obtained from the abdominal aorta 30 minutes after anesthesia with sodium pentobarbital (Somnopentyl, $64.8 \mathrm{mg} / \mathrm{ml}$, Kyoetsu Seiyaku Co. Ltd., Tokyo, Japan) diluted 5 times with saline $(65 \mathrm{mg} / \mathrm{kg}$, intramuscularly). Non-anticoagulated blood was mixed with saline (1:1). $3.6 \mathrm{ml}$ of the diluted blood and $0.4 \mathrm{ml}$ of potato filtrate or saline (control) (blood: filtrate $=9: 1$ ) were mixed in a syringe by inversion and the mixture was transferred into the GTT test tube.

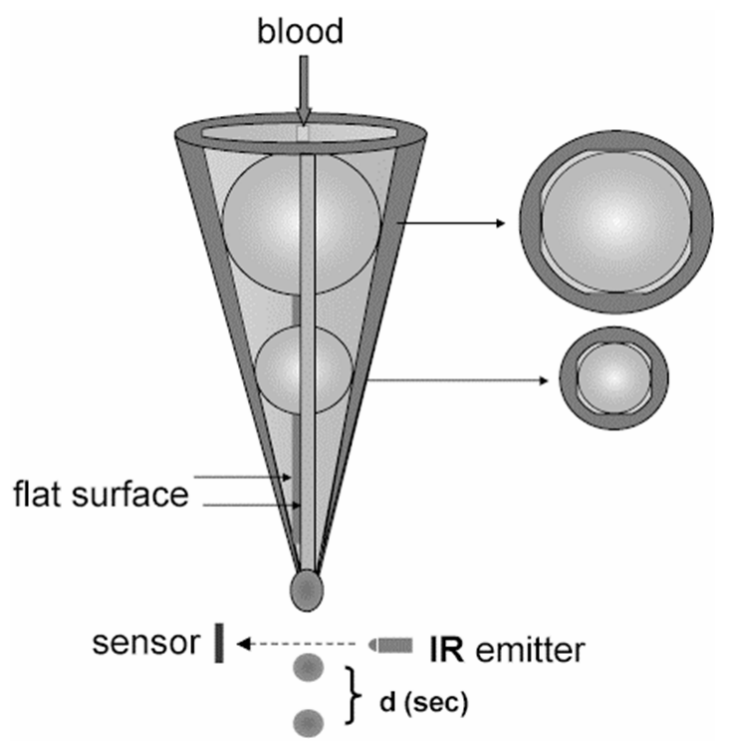

Figure 1. Schematic illustration of the GTT apparatus.

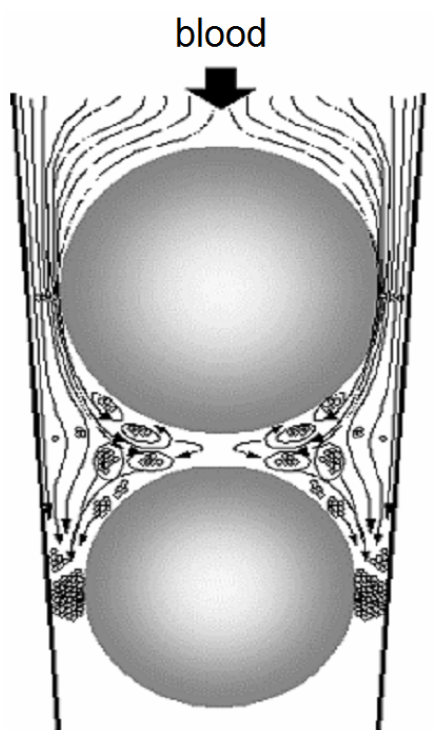

Figure 2. Schematic diagram showing the principle of the GTT. 


\subsection{In Vivo Assessment of Antithrombotic Effects Using Laser-Induced Thrombosis Test in Mice}

The He-Ne laser-induced platelet-rich thrombosis method has been previously described in detail $[17,18]$. Mice were anaesthetised with Somnopentyl $(65 \mathrm{mg} / \mathrm{kg}$, intramuscularly). A polyethylene tube (PE10, Becton Dickinson and Company, New Jersey, USA) was placed into the left femoral artery to inject dye and the carotid artery (450 - $500 \mu \mathrm{m}$ in diameter) was exposed by incision. The mouse was placed on a specially adapted microscope stage (Olympus Model BH-2, Olympus Co. Ltd., Tokyo, Japan) and Evans blue dye (30 mg/kg, Merck, Darnstadt, Germany) was injected intra-arterially. The centre of the exposed carotid artery was irradiated with a laser beam (Model Neo50MS, $25 \mathrm{~mW}$ under an objective lens, Neoark Co. Ltd., Osaka, Japan). Thrombus formation at the site of irradiation was monitored under epi-illumination and simultaneously recorded on videotape using a CCD camera (Model TMC-7, Takenaka System Co. Ltd., Kyoto, Japan).

\subsection{Oral Administration of Heated Potato Filtrates}

The vegetable filtrates or saline (control) were administered through a gastric tube in a volume of $30.8 \mathrm{ml} / \mathrm{kg}$ (heated filtrate). Half volume of the filtrate or saline (15.4 $\mathrm{ml} / \mathrm{kg}$ ) was repeatedly given at 30 minute interval as previously de-scribed [19]. The laser thrombosis experiment commenced $90 \mathrm{~min}$ after the second oral administration. Antithrombotic or prothrombotic effect was assessed by calculating the total thrombus size.

\subsection{Calculation of Thrombus Size}

Details of this technique have been described elsewhere [18]. Images of thrombus formation were computer-analysed at $10 \mathrm{sec}$ intervals. The area of thrombus was delineated and the mass of thrombus was calculated using Image $\mathrm{J}$ software (Image Processing and Analysis Java version 1.30, National Institutes of Health, Maryland, USA). Thrombotic status was expressed as the total sum of thrombus mass after the first 10 minutes of irradiation.

\subsection{Statistical Analysis}

Data from GTT were analysed by repeated ANOVA (General Linear Model), followed by the multiple comparison test (Dunnett), data from the laser-induced thrombosis experiments by the unpaired t-test. Values were expressed as means $\pm \mathrm{SEM}$. $\mathrm{P}<0.05$ was considered as the limit of significance. Analyses were performed using Statistical Package Unistat 5.6 Light (London, UK).

\section{RESULTS}

\subsection{Effects of Raw Filtrates of Various Potato Varieties on Shear-Induced Platelet Thrombosis and Spontaneous Thrombolysis in Vitro}

The results are shown in Table 1. The raw, undiluted filtrates from all varieties significantly inhibited platelet reactivity and four out of seven varieties maintained their inhibitory effect even after 3-fold dilution. As none of the filtrates affected the spontaneous thrombolytic activity (Lysis time), these potato filtrates exerted their antithrombotic effect by their anti-platelet effect.

Table 1. Effects of raw filtrates from seven potato varieties on shear-induced platelet thrombosis (occlusion time, OT) and spontaneous thrombolysis (lysis time, LT).

\begin{tabular}{|c|c|c|c|c|c|c|c|}
\hline \multicolumn{2}{|c|}{ Variety } & \multicolumn{2}{|c|}{ Dilution } & \multicolumn{2}{|c|}{ Occlusion time } & \multicolumn{2}{|c|}{ Lysis time } \\
\hline \multirow[t]{3}{*}{ Dejima } & saline & 344.7 & \pm & 51.8 & 1445.7 & \pm & 404.0 \\
\hline & $\times 1$ & 812.0 & \pm & $126.4^{* *}$ & & nd & \\
\hline & $\times 3$ & 513.8 & \pm & $81.5^{* *}$ & 2889.5 & \pm & 2175.7 \\
\hline \multirow[t]{3}{*}{ Cherie } & saline & 363.6 & \pm & 67.3 & 1207.5 & \pm & 191.2 \\
\hline & $\times 1$ & 820.9 & \pm & $165.1^{* *}$ & & nd & \\
\hline & $\times 3$ & 510.2 & \pm & $58.8^{*}$ & 1736.8 & \pm & 526.1 \\
\hline \multirow[t]{3}{*}{ Corolle } & saline & 335.8 & \pm & 30.8 & 1459.0 & \pm & 358.1 \\
\hline & $\times 1$ & 335.4 & \pm & $97.5^{* *}$ & & nd & \\
\hline & $\times 3$ & 335.6 & \pm & $66.4^{* *}$ & 1852.2 & \pm & 1955.3 \\
\hline \multirow[t]{3}{*}{ Cynthia } & saline & 359.2 & \pm & 35.1 & 1676.3 & \pm & 482.0 \\
\hline & $\times 1$ & 730.8 & \pm & $292.3^{* *}$ & & nd & \\
\hline & $\times 3$ & 544.0 & \pm & 86.8 & 998.6 & \pm & 217.4 \\
\hline \multirow[t]{3}{*}{ Sassy } & saline & 359.6 & \pm & 48.4 & 1305.0 & \pm & 367.6 \\
\hline & $\times 1$ & 714.6 & \pm & $87.3^{* *}$ & & nd & \\
\hline & $\times 3$ & 424.2 & \pm & 91.2 & 1683.3 & \pm & 852.4 \\
\hline \multirow[t]{3}{*}{ Chelsea } & saline & 359.3 & \pm & 78.2 & 1105.8 & \pm & 219.8 \\
\hline & $\times 1$ & 645.3 & \pm & $172.7^{* *}$ & & nd & \\
\hline & $\times 3$ & 398.2 & \pm & 48.5 & 2676.2 & \pm & 968.4 \\
\hline \multirow[t]{3}{*}{ Nishiyutaka } & saline & 293.5 & \pm & 54.2 & 1164.0 & \pm & 285.7 \\
\hline & $\times 1$ & 746.6 & \pm & $143.3^{* *}$ & & nd & \\
\hline & $\times 3$ & 475.3 & \pm & $113.4^{* *}$ & 1146.2 & \pm & 425.4 \\
\hline
\end{tabular}

${ }^{* *} \mathrm{P}<0.01,{ }^{*} \mathrm{P}<0.05, \mathrm{nd}:$ not determined. 
Table 2. Heat stability of antiplatelet activities of potato varieties harvested in autumn.

\begin{tabular}{|c|c|c|c|c|c|c|c|}
\hline \multicolumn{2}{|c|}{ Variety } & \multicolumn{2}{|c|}{ Dilution } & \multicolumn{2}{|c|}{ Occlusion time } & \multicolumn{2}{|c|}{ Lysis time } \\
\hline Dejima & saline & 333.2 & \pm & 41.8 & 1425.0 & \pm & 235.2 \\
\hline & $\times 1$ & 761.8 & \pm & $87.1^{* *}$ & & nd & \\
\hline & $\times 3$ & 451.6 & \pm & $71.1^{*}$ & 1771.7 & \pm & 468.4 \\
\hline \multirow[t]{3}{*}{ Cherie } & saline & 355.6 & \pm & 69.5 & 1426.2 & \pm & 361.8 \\
\hline & $\times 1$ & 626.5 & \pm & $160.7^{* *}$ & 1091.2 & \pm & 1149.2 \\
\hline & $\times 3$ & 357.5 & \pm & 60.2 & 1275.4 & \pm & 346.7 \\
\hline \multirow[t]{3}{*}{ Corolle } & saline & 367.1 & \pm & 27.9 & 1336.2 & \pm & 275.6 \\
\hline & $\times 1$ & 783.7 & \pm & $76.2^{* *}$ & & nd & \\
\hline & $\times 3$ & 410.5 & \pm & 46.1 & 1466.3 & \pm & 188.1 \\
\hline \multirow[t]{3}{*}{ Nishiyutaka } & saline & 373.5 & \pm & 40.9 & 1387.8 & \pm & 420.1 \\
\hline & $\times 1$ & 528.4 & \pm & $108.1^{* *}$ & & nd & \\
\hline & $\times 3$ & 367.4 & \pm & 64.8 & 1285.2 & \pm & 380.1 \\
\hline
\end{tabular}

${ }^{* *} \mathrm{P}<0.01,{ }^{*} \mathrm{P}<0.05$, nd: not determined.

\subsection{Heat Stability of Antithrombotic Activity}

Heat stability of filtrates from four varieties, Dejima, Cherie, Corolle and Nishiyutaka, which were antiplatelet reactive at three-fold dilution in Table 1 was investigated. Results are shown in Table 2. Dejima was the most heatresistant while antiplatelet reactivity of other potatoes was reduced.

\subsection{Antithrombotic Activity of Heated Filtrate of Nishiyutaka after Oral Administration}

As in Table 2, heated Dejima filtrate showed the strongest antithrombotic activity after oral administration (Figure 3).

\section{DISCUSSION}

Thrombotic diseases are one of the leading causes of deaths. Finding a reliable test(s) to detect thrombotic tendency and monitor the effect of antiplatelet medication would be critically important. It is widely appreciated, that thrombus formation in vivo is governed by interactions between blood flow, blood components and the blood vessel, as proposed by Virchow. Unfortunately the pathophysiological relevance of tests presently in use to assess thrombotic status is weak.

Novel experimental techniques tests, which use non-anticoagulated blood and shear forces as platelet agonists have been proposed $[12,13,16]$. Clinical and experimenttal studies have supported that these tests may be more relevant in assessing thrombotic status in vivo [12-16], that those using anticoagulated blood. In our present and earlier studies we used such methods to test various fruits and vegetables for antithrombotic effect [14,15,19-21]. Earlier, we identified specific antithrombotic potato varieties produced in the spring and summer [21]. The present study tested seven potato varieties harvested in the

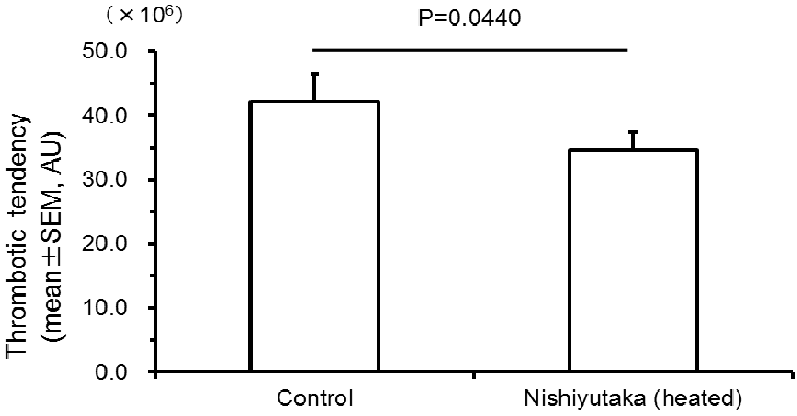

Figure 3. Antithrombotic activity of a potato variety harvested in autumn, Nishiyutaka (heated filtrate).

autumn. Out of the four active varieties, one Nishiyutakashowed significant anti-platelet effect and the effect was still preserved after heat-treatment of the extract.

\section{CONCLUSION}

Seven varieties of autumn harvested potatoes were tested for experimental antithrombotic effect. One variety (Nishiyutaka) showed antithrombotic effect not just in vitro, but also after oral administration, in vivo. As potato is one of the basic foods, potentially this potato variety could be a useful component of an antithrombotic diet. Further tests are planned to test Nishiyutaka extract in human blood in vitro and, should it be still antithrombotic, in human volunteers.

\section{REFERENCES}

[1] Lichtenstein, A.H., Appel, L.J., Brands, M., Carnethon, M., Daniels, S., Franch, H.A., Franklin, B., Kris-Etherton, P., Harris, W.S., Howard, B., Karanja, N., Lefevre, M., Rudel, L., Sacks, F., Van Horn, L., Winston, M. and Wylie-Rosett, J. (2006) Summary of American heart association diet and lifestyle recommendations revision. Arteriosclerosis, Thrombosis and Vascular Biology, 26, 
2186-2191. doi:10.1161/01.ATV.0000238352.25222.5e

[2] Ulbricht, T.L. and Southgate, D.A. (1991) Coronary heart disease: Seven dietary factors. Lancet, 338, 985-992. doi:10.1016/0140-6736(91)91846-M

[3] Renaud, S. and De Lorgeril, M. (1992) Wine, alcohol, platelets, and the French paradox for coronary heart disease. Lancet, 339, 1523-1526. doi:10.1016/0140-6736(92)91277-F

[4] Joshipura, K.J., Ascherio, A., Manson, J.E., Stampfer, M.J., Rimm, E.B., Speizer, F.E., Hennekens, C.H., Spiegelman, D. and Willett, W.C. (1999) Fruit and vegetable intake in relation to risk of ischemic stroke. Journal of the American Medical Association, 282, 1233-1239. doi:10.1001/jama.282.13.1233

[5] Liu, S., Manson, J.E., Lee, I.M., Cole, S.R., Hennekens, C.H., Willett, W.C. and Buring, J.E. (2000) Fruit and vegetable intake and risk of cardiovascular disease: The women's health study. American Journal of Clinical Nutrition, 72, 922-928.

[6] Joshipura, K.J., Hu, F.B., Manson, J.E., Stampfer, M.J., Rimm, E.B., Speizer, F.E., Colditz, G., Ascherio, A., Rosner, B., Spiegelman, D. and Willett, W.C. (2001) The effect of fruit and vegetable intake on risk for coronary heart disease. Annals of the Internal Medicine, 134, 11061114.

[7] Bazzano, L.A., He, J., Ogden, L.G., Loria, C.M., Vupputuri, S., Myers, L. and Whelton, P.K. (2002) Fruit and vegetable intake and risk of cardiovascular disease in US adults: The first national health and nutrition examination survey epidemiologic follow-up study. American Journal of the Clinical Nutrition, 76, 93-99.

[8] Harrison, P. (2005) Platelet function analysis. Blood Review, 19, 111-123. doi:10.1016/j.blre.2004.05.002

[9] Ferreiro, J.L., Sibbing, D. and Angiolillo, D.J. (2010) Platelet function testing and risk of bleeding complications. Thrombosis et Haemostasis, 103, 1128-1135. doi:10.1160/TH09-11-0799

[10] Lippi, G., Cervellin, G., Franchini, M. and Favaloro, E.J. (2010) Biochemical markers for the diagnosis of venous thromboembolism: The past, present and future. Journal of the Thrombosis and Thrombolysis, 30, 459-471. doi:10.1007/s11239-010-0460-x

[11] Gorog, D.A., Sweeny, J.M. and Fuster, V. (2009) Antiplatelet drug "resistance". Part 2: Laboratory resistance to antiplatelet drugs-fact or artifact? Nature Reviews Cardiology, 6, 365-373.

[12] Ratnatunga, C.P., Edmondson, S.F., Rees, G.M. and
Kovacs, I.B. (1982) High-dose aspirin inhibits shear-induced platelet reaction involving thrombin generation. Circulation, 85, 1077-1082.

[13] Gorog, D.A. and Kovacs, I.B. (1995) Thrombotic status analyser. Thrombosis et Haemostasis, 73, 514-520.

[14] Yamamoto, J., Naemura, A., Ura, M., Ijiri, Y., Yamashita, T., Kurioka, A. and Koyama, A. (2006) Testing various fruits for anti-thrombotic effect: I. Mulberries. Platelets, 17, 555-564. doi:10.1080/09537100600759295

[15] Yamamoto, J., Naemura, A., Ijiri, Y., Ogawa, K., Suzuki, T., Shimada, Y., Giddings, J.C. (2008) The antithrombotic effects of carrot filtrates in rats and mice. Blood Coagulation \& Fibrinolysis, 19, 785-792. doi:10.1097/MBC.0b013e3283177b19

[16] Yamamoto, J., Yamashita, T., Ikarugi, H., Taka, T., Hashimoto, M., Ishii, H., Watanabe, S. and Kovacs, I.B. (2003) Gorog thrombosis test: A global in-vitro test of platelet function and thrombolysis. Blood Coagulation \& Fibrinolysis, 14, 31-39. doi:10.1097/00001721-200301000-00007

[17] Kovacs, I.B., Tigyi-Sebes, A., Trombitas, K. and Gorog, P. (1975) Evans blue: An ideal energy-absorbing material to produce intravascular microinjury by $\mathrm{He}-\mathrm{Ne}$ gas laser. Microvascular Research, 10, 107-124. doi:10.1016/0026-2862(75)90025-4

[18] Ijiri, Y., Miura, M., Hashimoto, M., Fukunaga, C., Wata-nabe, S., Kubota, A., Oiwa, K., Okuda, T. and Yamamoto, J. (2002) A new model to evaluate the diet-induced prothrombotic status, using $\mathrm{He}-\mathrm{Ne}$ laser-induced throm-bogenesis in the carotid artery of apolipoprotein E-deficient and low-density lipoprotein receptor-deficient mice. Blood Coagulation \& Fibrinolysis, 13, 497-504. doi:10.1097/00001721-200209000-00004

[19] Yamamoto, J., Taka, T., Yamada, K., Ijiri, Y., Murakami, M., Hirata, Y., Naemura, A., Hashimoto, M., Yamashita, T., Oiwa, K., Seki, J., Suganuma, H., Inakuma, T. and Yoshida, T. (2003) Tomatoes have natural antithrombotic effects. British Journal of Nutrition, 90, 1031-1038. doi:10.1079/BJN2003994

[20] Naemura, A., Mitani, T., Ijiri, Y., Tamura, Y., Yamashita, T., Okimura, M. and Yamamoto, J. (2005) Anti-thrombotic effect of strawberries. Blood Coagulation \& Fibrinolysis, 16, 501-509. doi:10.1097/01.mbc.0000184737.50594.a8

[21] Ichiura, D., Naemura, A., Ura, M., Mori, M. and Yamamoto, J. (2009) Antithrombotic effect of potato in animal experiments. Food, 3, 8-12. 